\title{
Reforming perpendicular shocks in the presence of pickup protons: initial ion acceleration
}

\author{
R. E. Lee ${ }^{1}$, S. C. Chapman ${ }^{1,2}$, and R. O. Dendy ${ }^{1,3}$ \\ ${ }^{1}$ Space and Astrophysics Group, Department of Physics, University of Warwick, Coventry CV4 7AL, UK \\ ${ }^{2}$ Radcliffe Institute for Advanced Study, Harvard University, USA \\ ${ }^{3}$ UKAEA Culham Division, Culham Science Centre, Abingdon, Oxfordshire, OX14 3DB, UK
}

Received: 03 August 2004 - Revised: 3 December 2004 - Accepted: 6 December 2004 - Published: 28 February 2005

\begin{abstract}
Acceleration processes associated with the heliospheric termination shock may provide a source of anomalous cosmic rays (ACRs). Recent kinetic simulations of supercritical, quasi-perpendicular shocks have yielded time varying shock solutions that cyclically reform on the spatiotemporal scales of the incoming protons. Whether a shock solution is stationary or reforming depends upon the plasma parameters which, for the termination shock, are ill defined but believed to be within the time-dependent regime. Here we present results from high phase space resolution particlein-cell simulations for a three-component plasma (solar wind protons, electrons and pickup protons) appropriate for the termination shock. We find reforming shock solutions which generate suprathermal populations for both proton components, with the pickup ions reaching energies of order twenty times the solar wind inflow energy. This suprathermal "injection" population is required as a seed population for subsequent acceleration at the shock which can in turn generate ACRs.
\end{abstract}

Key words. Interplanetary physics (Cosmic rays, Heliopause and solar wind termination) - Space plasma physics (Shock waves)

\section{Introduction}

With the approach of the Voyager spacecraft towards the heliopause and in particular the termination shock (Burlaga et al., 2003; Gurnett et al., 2003), together with possible crossings thereof (Krimigis et al., 2003; McDonald et al., 2003), the problem of the acceleration of anomalous cosmic rays (ACRs) (Fisk et al., 1974; Pesses et al., 1981) in the outer heliosphere is at the forefront of current research (see, for example, Kucharek and Scholer, 1995; Zank et al., 1996; Rice et al., 2000; Zank et al., 2001; Lever et al., 2001). This provides an opportunity for comparing theoretical results with observations.

Correspondence to: R. E. Lee

(leer@astro.warwick.ac.uk)
Kinetic simulations are a useful tool to investigate, selfconsistently, local acceleration processes at the shock. Particle-in-cell (PIC) simulations of quasi-perpendicular shocks (see, for example, Biskamp and Welter, 1972; Lembège and Dawson, 1987; Lembège and Savoini, 1992; Shimada and Hoshino, 2000; Schmitz et al., 2002a; Hada et al., 2003; Lee et al., 2004a), have found that, for a certain range of parameters, the shock solutions are not static, rather reforming on the gyro scales of the incoming bulk protons. Hybrid simulations, on the other hand, typically generate static solutions (see, for example, Burgess et al., 1989; Kucharek and Scholer, 1995; Lipatov and Zank, 1999), although reforming solutions are recovered for a range of values of resistivity (Quest, 1985; Hellinger et al., 2002). The time dependent electromagnetic fields at reforming shocks have been found to accelerate a fraction of the bulk proton population to suprathermal energies (Lee et al., 2004a), and this is a possible cosmic ray injection mechanism at supernova remnants. Parameters relevant to the heliospheric termination shock, although ill defined, are consistent with those expected to yield reforming solutions in a quasiperpendicular geometry. The use of a quasi-perpendicular geometry is appropriate if we make the assumption that Parker's solar wind model is still valid in the outer heliosphere. Following Pesses et al. (1981) one concludes that the shock is close to perpendicular (the angle between magnetic field and shock normal $>80^{\circ}$ ) at heliographic latitudes less than $75^{\circ}$. Pioneer 11 measurements show the existence of two broad distributions of shock propagation angle, centred on $90^{\circ}$ and $270^{\circ}$, with half widths of $25^{\circ}$ (Smith, 1993).

Importantly, the heliospheric termination shock is generated in the presence of pickup ions originating from the transheliopause interstellar medium. Two questions then arise, which we address here: first, whether the reforming shock solutions found previously persist with the self consistent inclusion of a pickup ion population; and second, to what extent are both solar wind and pickup protons accelerated in the resulting time dependent electromagnetic fields. 


\section{Simulation}

We use a 1.5 -D $(1 x, 3 v)$ relativistic electromagnetic PIC code to simulate the structure and evolution of a supercritical, collisionless, perpendicular magnetosonic shock. Simulations of reforming shocks in quasi-perpendicular and pureperpendicular reforming shocks have been performed, for example, by Scholer et al. (2003), where it is shown that the additional wavemodes supported by oblique geometry do not strongly affect the overall structure and dynamics of the reforming shock. In a PIC simulation, the distribution functions of all particle species are represented by computational super-particles, the phase space locations of which are evolved via the Lorentz force law, whilst the electromagnetic fields are defined on a spatial grid and evolved via the full Maxwell equations (see, for example, Birdsall and Langdon, 1991). The simulation setup is that described in Lee et al. (2004a), with the addition of pickup protons, the results of which are presented for the first time here. We find reforming shock solutions in this 1.5-D geometry, where scalar and all three components of vector quantities are functions of one spatial coordinate and time. Reforming shock solutions have also been found in higher dimensions (Lembège and Savoini, 1992).

To accurately reproduce the effects of pickup protons, we simulate three species within our code: electrons, solar wind protons, and pickup protons. Particles are injected on the left-hand side of the simulation box with a drift speed $v_{\text {inj. }}$. The simulation is conducted in the downstream rest frame, so if $v_{1}$ is the upstream and $v_{2}$ the downstream flow speed derived from the Rankine-Hugoniot relations, $v_{\text {inj }}$ can be defined from the Alfvénic Mach number, $M_{A}$ by: $v_{\text {inj }}=M_{A} v_{A}-v_{2}$. For solar wind protons and electrons, each individual particle has its initial speed modified by an additional Maxwellian random velocity, chosen from a thermal distribution characterised by a $v_{\text {thm }}$ for each species. Pickup protons are born with similar values of perpendicular velocity and arbitrary gyrophase, centred on the drift speed. The pickup protons are modelled by a population with velocities in the form of a spherical shell, centred on $v_{\text {inj }}$, radius $v_{1}$. On the particle injection boundary, the magnetic field $\left(B_{z, 1}\right)$ is constant and the electric field $\left(E_{y, 1}\right)$ is calculated self consistently. We use the piston method to generate the shock, and shock-following algorithms, to obtain a sufficiently long run time, as described in Schmitz et al. (2002a).

Here results are presented from simulations of a perpendicular shock propagating into a collisionless plasma, at an Alfvénic Mach number $\left(M_{A}\right)$ of 8 . The upstream ratio of plasma thermal pressure to magnetic field pressure for the solar wind thermal protons is taken as $\beta_{i}=0.2$, and for the electrons $\beta_{e}=0.5$. The pickup proton effective temperature is characterised by $v_{1}$ due to their initial shell distribution (after Kucharek and Scholer, 1995; Zank et al., 1996; Ellison et al., 1999). To enable ion and electron time scales to be captured within the same simulation, with reasonable computational overheads, it is necessary to reduce the simulation mass ratio for ions and electrons to $M_{R}=m_{i} / m_{e}=20$, and the ratio of electron plasma frequency to electron cyclotron frequency $\omega_{p e} / \omega_{c e}=20$ (in common with Shimada and Hoshino, 2000; Schmitz et al., 2002a,b; Lee et al., 2004a). Simulations at higher mass ratios have been conducted by, for example, Lembège and Savoini (2002) at $M_{R}=400$, and Scholer et al. (2003) at $M_{R}=1840$; however, to achieve reasonable runtimes and simulation domains, a ratio of $\omega_{p e} / \omega_{c e}=[2, \sqrt{8}]$ was used, for example, in the latter. In our simulations a lower mass ratio is chosen to allow for sufficient resolution of the ion phase space to study the ion dynamics. Scholer et al. (2003) demonstrate that reformation is a low $\beta$ process, and is not an artifact of unrealistic mass ratios, and that the differences that occur between simulations at $M_{R}=20$ and $M_{R}=1840$ are in the nature of the instabilities in the foot region. Previous work suggests (see, Lee et al. 2004a, Lee et al. 2004b) that the reflected ion dynamics are insensitive to these instabilities, and importantly we resolve the same length scales for the foot region $\left(\sim \lambda_{c i}\right)$ and shock ramp (of the order of $\left.c / \omega_{p e}\right)$, thus we expect our conclusions to hold at $M_{R}=1840$.

\section{Results}

The key phenomenology found in our PIC simulations arises from the non-time-stationary nature of the reforming shock. This is shown in Figs. 1 and 2; these plot magnetic field strength and $\int E_{x} d x$ as functions of $x$ and time for two simulations, on the left, for solar wind protons only and on the right, with the addition of a pickup proton population at $10 \%$ of the density of the background. As with all figures in this paper, they are presented in a frame where the downstream plasma is at rest, using data collected from a time segment of the simulation when the shock has formed and is propagating independently of the boundary conditions. Units are normalised to upstream parameters for the solar wind protons, thus $\lambda_{c i}$ is the upstream solar wind proton cyclotron radius, $\omega_{c i}$ is the upstream solar wind proton cyclotron frequency, and $\mathcal{E}_{\mathrm{inj}, \mathrm{SW}}=m_{i} v_{\mathrm{inj}}^{2} / 2$ is the solar wind proton injection energy. The shock can be seen to move upstream, from the right to the left of the simulation box, over time, with peaks in magnetic field (the shock ramp) recurring, and the potential well expanding and collapsing, on the time scale of the local proton cyclotron period. Over the course of each cycle, a stationary shock ramp forms at the turnaround point of protons in the foot region. Then a new foot region extends into the upstream region as protons reflect from this new shock front. A new ramp (magnetic field peak) then starts to form at the upstream edge of this foot region, and another cycle begins. The last of the original foot region protons gyrate into the downstream plasma as the new shock starts to reflect a new foot region population. The shock thus propagates upstream (left) in a stepwise fashion. Comparison of a shock simulation containing no pickup ions (panel A in Figs. 1 and 2, and see also, Lembège and Savoini, 1992; Shimada and Hoshino, 2000; Schmitz et al., 2002a; Lee et al., 2004a) to a shock simulation containing $10 \%$ pickup protons (panel B 

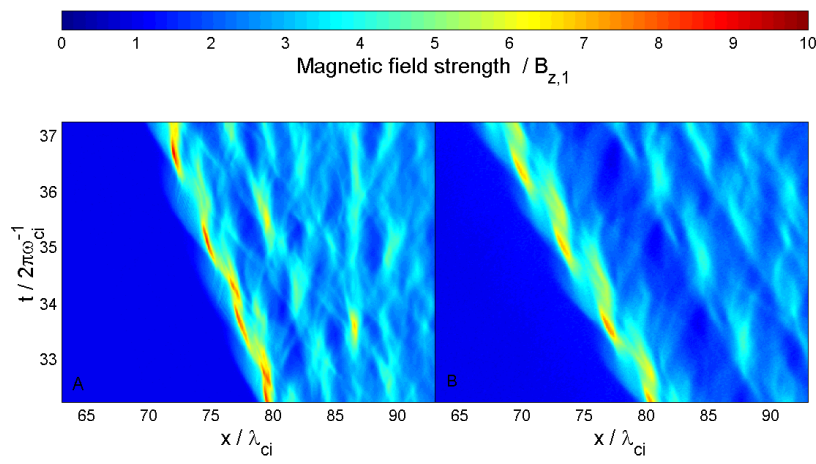

Fig. 1. Evolution of perpendicular magnetic field strength, over time (vertical axis), and space (horizontal axis), for a simulation in the absence of pickup ions (panel A) and a simulation containing $10 \%$ pickup ions (panel B).
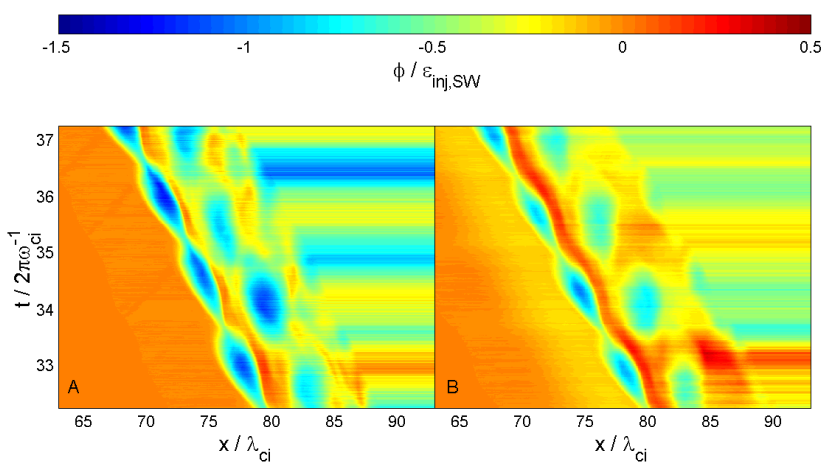

Fig. 2. Evolution of $\phi=\int E_{x} d x$, over time (vertical axis), and space (horizontal axis). In the absence of pickup protons (A), and with $10 \%$ pickup protons (B).

in Figs. 1 and 2), shows that the reformation spatio-temporal scales are similar.

Figure 3 shows a snapshot of the spatial distributions of $x$ velocity and kinetic energy for both proton species, together with $\int E_{x} d x$, and perpendicular magnetic field, at the beginning of a reformation cycle $\left(t=34.5 \times 2 \pi \omega_{c i}^{-1}\right)$. The temporal evolution of both proton species in phase space over a reformation cycle is shown in Fig. 4, together with the corresponding scalar $\int E_{x} d x$ and perpendicular magnetic field in Fig. 5. From this figure we see that the addition of $10 \%$ pickup protons does, however, lead to an extended foot region, observable by the slow decline into the shock's potential structure starting $\sim 6 \lambda_{c i}$ upstream, and in the downstream acts to suppress magnetic field fluctuations over a distance of $\sim 5 \lambda_{c i}$ downstream of the shock front.

In Fig. 3 protons from both species flow in from the lefthand, injection boundary. Solar wind protons continue until they reach the shock front (currently situated at $\sim 75 \lambda_{c i}$ ). At this stage of the reformation cycle, the shock front is almost stationary (see Fig. 1), so that a fraction of the solar wind protons specularly reflect, in the downstream rest frame, from the ramp in the magnetic field and its accompanying potential, with $v_{x} \rightarrow-v_{x}$ (panel 5). The reflected solar wind pro-

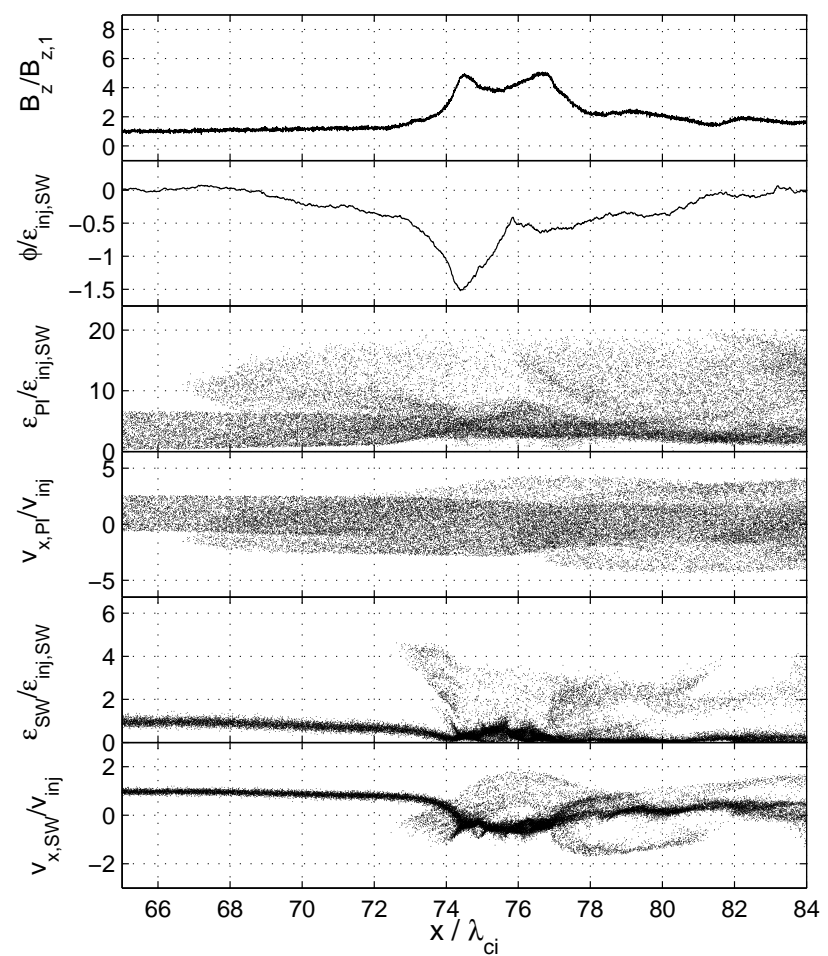

Fig. 3. Spatial cross section of the simulation in the shock region at $t=34.5 \times 2 \pi \omega_{c i}^{-1}$. The top panel (panel 1) shows perpendicular magnetic field strength, $B_{z}$, normalised to the upstream value, $B_{z, 1}$. Panel 2 shows $\phi=\int E_{x} d x$ normalised to the solar wind proton in-

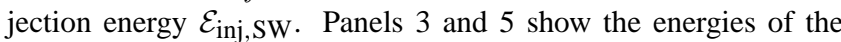
pickup protons and the solar wind protons, respectively, again nor-

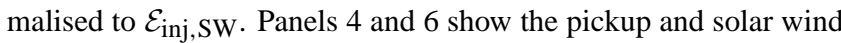
proton $x$-velocities, normalised to the injection velocity $v_{\text {inj }}$.

tons move upstream to form a relatively energetic population, as shown in panel 4 , gaining energies dispersed from

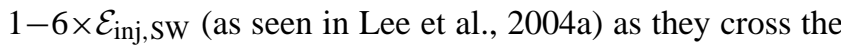
shock front and move into the downstream region.

Pickup protons also flow in until they reach the shock front, at which stage they, too, are reflected (panel 3). The presence of pickup protons leads to an extended foot region with two components: the reforming foot region associated with the solar wind protons close to the shock, plus a weak foot region extending up to $\sim 6 \lambda_{c i}$ upstream. The relative spatial scales of these foot region populations may be understood in terms of specular reflection at the shock ramp. If the protons simply reflect specularly, that is, reverse their component of velocity normal to the shock front $\left(v_{n}\right)$ as they encounter the ramp, then the length-scale for each population is $L_{\text {foot }}=\left|v_{n}\right| / \omega_{c i}$. This is governed by $v_{n}=v_{\text {inj }}+_{\text {thm }} \sin \left(\omega_{c i} t+\phi\right)$ : for thermal protons this implies $v_{n} \simeq v_{\text {inj }}$ because $v_{\text {thm }, \mathrm{SW}} \ll v_{\text {inj }}$; whereas for the pickup protons $v_{\text {thm,PI }} \simeq v_{\text {inj }}$, so that $0<v_{n}<2 v_{\text {inj }}$. Thus, after specular reflection, the pickup protons create an extended foot region by moving further back into the upstream region, with a corresponding larger excursion in $y$. 


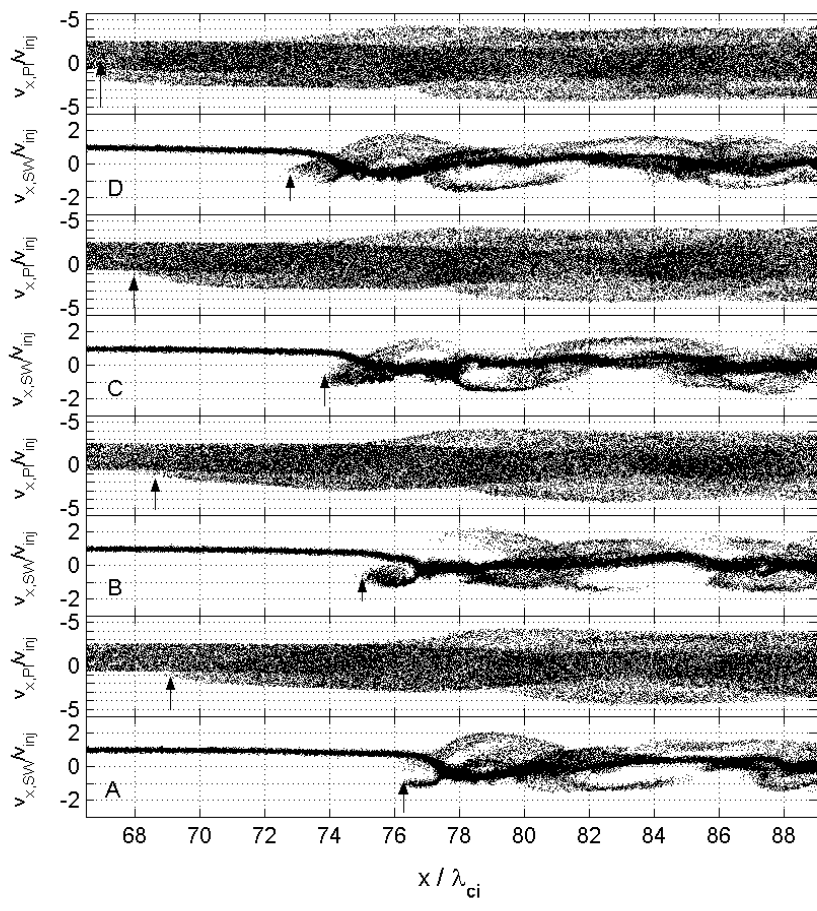

Fig. 4. Phase space $\left(v_{x} / v_{\text {inj }}\right.$ vs. $\left.x / \lambda_{c i}\right)$ for solar wind protons (lower panel of each pair) and pickup protons (upper panels) in the shock region. Four pairs of panels at equal time intervals of $0.4 \times 2 \pi \omega_{c i}^{-1}$, starting at time $t=33.3 \times 2 \pi \omega_{c i}^{-1}$ (lowest two panels). The maximum upstream position for the reflected ions in each species are indicated for each snapshot.

The evolution of the phase space of both proton species over the course of a reformation cycle is shown in Fig. 4; this is accompanied by Fig. 5 which shows the magnetic field and $\int E_{x} d x$ at the same times. Visible in $\int E_{x} d x$ in Fig. 5 is the weak potential feature, associated with the pickup protons, that extends $\sim 6 \lambda_{c i}$ upstream of the potential feature associated with the solar wind protons. This extended potential changes little over a reformation cycle, particularly when compared to the changes visible in the potential associated with reflecting solar wind protons. The maximum upstream positions for reflected pickup protons and solar wind protons are indicated by arrows on Fig. 4 and on the $\int E_{x} d x$ panels in Fig. 5, to highlight this point. The presence of an extended foot region is also visible to a lesser degree in the magnetic field profiles; these also clearly show the nontimestationary nature of the shock ramp. When it is possible to do so (panels A, C and D) we observe a shock ramp thickness of the order of a few electron inertial lengths (in common with Lembège and Savoini, 1992; Scholer et al., 2003). This value of the shock thickness can also be seen in Fig. 6 which shows magnetic field strength and $\int E_{x} d x$ profiles for a simulation in the absence of pickup protons, at four times over the course of a reformation cycle comparable to those shown in Fig. 5. Comparing Figs. 5 and 6 shows $\int E_{x} d x$ and magnetic field structure associated with the bulk solar wind protons, that are qualitatively similar to that arising in the presence of pickup protons.

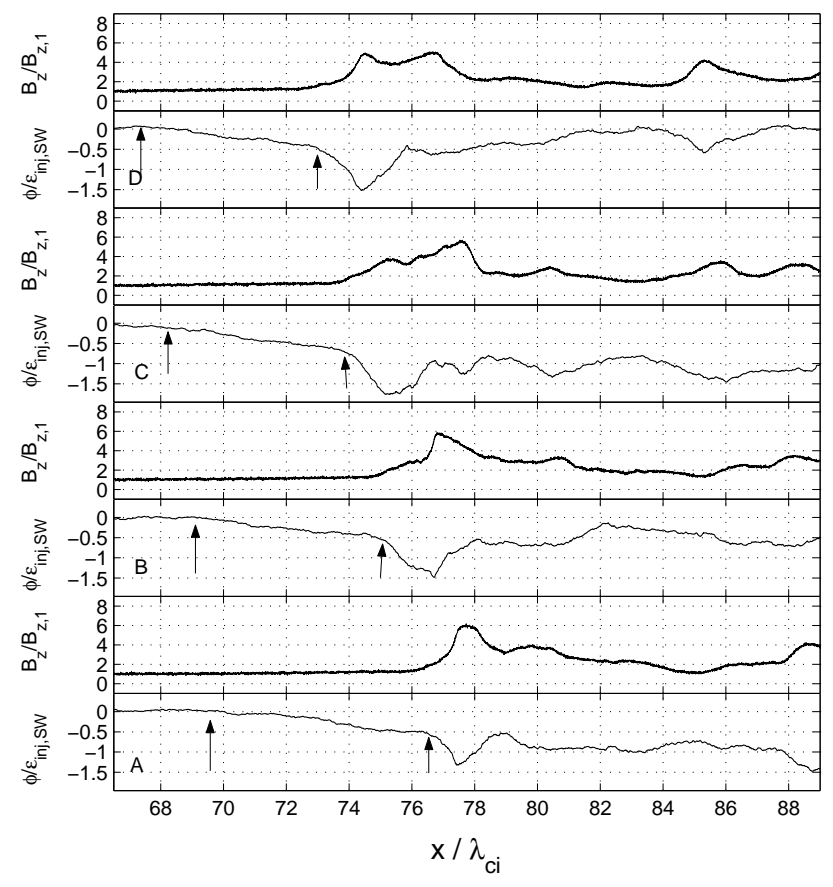

Fig. 5. Four pairs of panels, corresponding to the spatio-temporal coordinates of the panels in Fig. 4, showing perpendicular magnetic field strength (upper panel of each pair), and $\phi=\int E_{x} d x$ (lower panels) in the shock region. As in Fig. 4, the maximum upstream positions of reflected protons for each species are indicated, with the furthest upstream arrow relating to the pickup ion species.

From the magnetic field profiles it can be seen that the field is $B_{z} / B_{z, 1} \sim 3$, on average, in the downstream region. Taking the average time between minima in the shock's foot region value of $\int E_{x} d x$, the reformation time is $\sim 1.5 \times 2 \pi \omega_{c i}^{-1}$ in the simulations with both $0 \%$ and $10 \%$ pickup protons, as can be seen in Fig. 2. In terms of the average field just downstream of the overshoot, the reformation time is then $\sim 0.5$ local ion gyroperiods.

The differences in temporal evolution for the two species can be seen when the counts of reflected protons are compared. We count the number of protons upstream of the minima in $\int E_{x} d x$ with, in the case of solar wind protons, velocities directed away from the shock front and in the case of the pickup protons, those protons with velocities outside of the injected shell in velocity space. The time variation of these reflected populations is shown in Fig. 7. In contrast to the solar wind protons, the pickup proton reflected ion count (and consequently foot region structure) is stable over a reformation cycle, with slight changes coinciding with times of changing position for the minima in $\int E_{x} d x$ as the reforming shock advances. The solar wind proton reflection count, on the other hand, varies by a factor of $\sim 2.5$ over the course of a reformation cycle.

To understand the energy gain processes, it is useful to consider the evolution of $\int \mathbf{F} \cdot \mathbf{v} d t$, and its components, calculated along proton trajectories. Specifically, in Fig. 8 we examine a solar wind proton that reaches $\sim 6 \mathcal{E}_{\text {inj,SW }}$, and a 


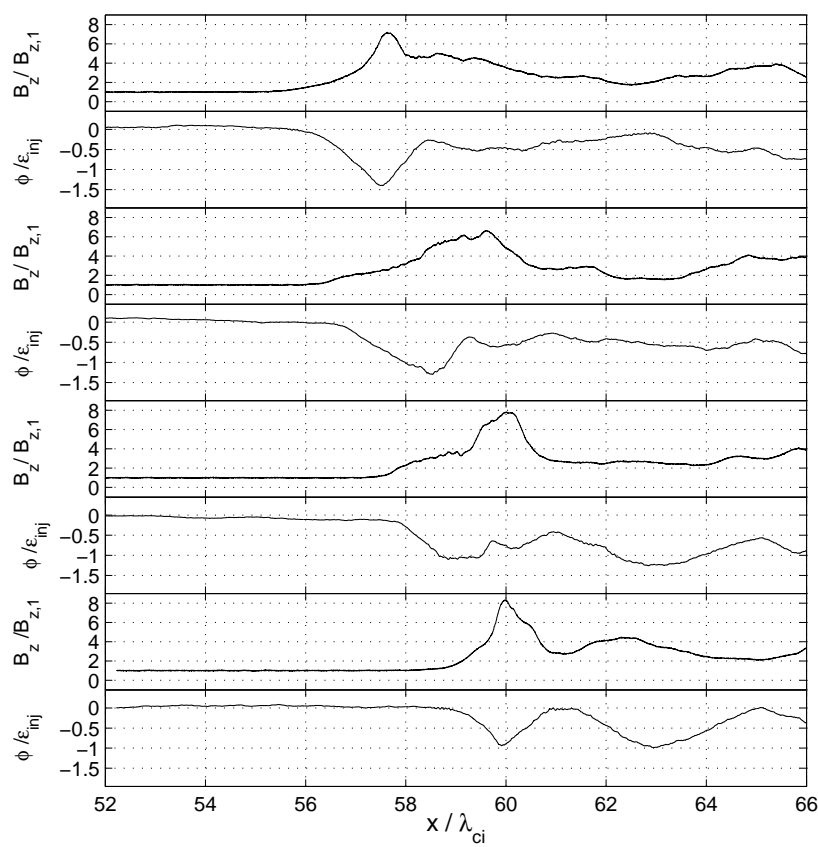

Fig. 6. Four pairs of panels, showing perpendicular magnetic field strength (upper panel of each pair), and $\phi=\int E_{x} d x$ (lower panels) in the shock region for a simulation in the absence of pickup ions. The panels are separated by equal time intervals of $0.4 \times 2 \pi \omega_{c i}^{-1}$, starting at time $t=33.3 \times 2 \pi \omega_{c i}^{-1}$ (lowest two panels).

pickup proton that journeys $\sim 8 \lambda_{c i}$ upstream after reflection, before returning and reaching high energy on crossing the shock front. To relate these changes to the shock dynamics, the proton trajectories are superimposed upon the spatiotemporally evolving $\int E_{x} d x$ in the bottom panel of Fig. 8 . The corresponding local $\int E_{x} d x$ experienced by these two protons is plotted in the middle panel of Fig. 8. Detailed analysis of the energetics of the solar wind protons (Lee et al., 2004a) suggests that the electromagnetic fields relevant to acceleration to suprathermal energies are on ion scales. As discussed in Lee et al. (2004a,b), for example, the fluid equations treated appropriately lead to

$E_{x, i} \simeq-\frac{1}{e n \mu_{0}} \frac{\partial\left(B_{z}^{2} / 2+B_{y}^{2} / 2\right)}{\partial x}-v_{i, y} B_{z} ;$

defining the bulk potential in terms of integral $E_{\text {xidx }}$, then removes features, such as electron phase space holes, but maintains ion scale features, such as the foot region potential structure. The rate of change of energy $e \mathbf{E} \cdot \mathbf{v}$ integrated over the trajectories of energetic ions with the electric field from Eq. (1) is then found to track that calculated using the full electric field from the PIC simulation. Previous PIC simulations for a range of values of $m_{i} / m_{e}$ (see, for example, Lembège and Savoini, 1992; Scholer et al., 2003) show a variety of kinetic instabilities in the foot region, and in our results, features, possibly related to the Buneman instability, are visible in the foot region of the solar wind ion phase space in Figs. 3 and 4. These features occur on elec-

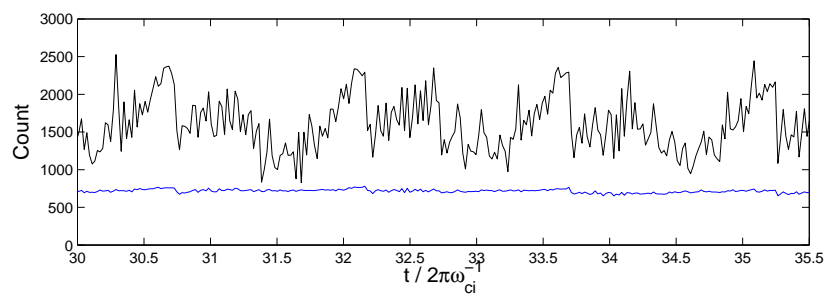

Fig. 7. Reflection rates over a series of reformation cycles. Solar wind protons are in black, and pickup protons in blue. Counts are of particles upstream of the minima in $\int E_{x} d x$ associated with the shock front, with velocities, in the case of solar wind ions directed in the direction of shock propagation, or for pickup ions, outside of the initial injected spherical distribution. Pickup ion counts are normalised to solar wind counts.

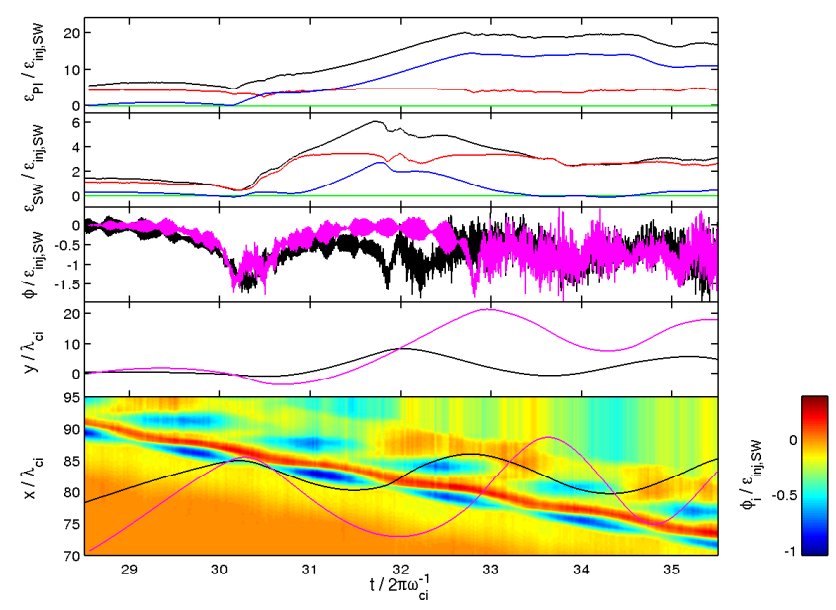

Fig. 8. Top panel (panel 1) shows the energy of a pickup proton, calculated from $\int \mathbf{F} \cdot \mathbf{v} d t$ and its components $(x$-red, $y$-blue, $z$-green, total-black), panel 2 the energy of a solar wind proton. Panel 3 shows $\int E_{x} d x$ at the position of both protons (solar wind-black, pickup-purple), panel 4 the $y$-positions, calculated from $y=\int v_{y} d t$, and panel 5 the $x$-positions overlayed onto the shock potential on ion scales (plotted as $\int E_{x, i} d x$ ).

tron spatio-temporal scales, and here we simply note that, as shown in Lee et al. (2004b), the ion dynamics will, from the above argument, be insensitive to structures on electron kinetic scales.

The proton trajectories in Fig. 8 display both similarities and differences during their shock interactions. Both particles encounter the shock when the magnetic field is at its strongest (Fig. 1), and the potential at its narrowest (panel 5). They are reflected off the shock front, moving upstream into the foot region gaining energy (panels 1 and 2) in the local time dependent electric field. Their gyration and the propagation of the shock then brings them back towards the shock front, where their energies are too high for a second reflection, and they enter the downstream region (panel 5).

The $x$-components of $\int \mathbf{F} \cdot \mathbf{v} d t$ for both protons are similar (red, panels 1 and 2 of Fig. 8). Initially, as the protons move towards the shock, there is an $x$-energy loss of roughly 


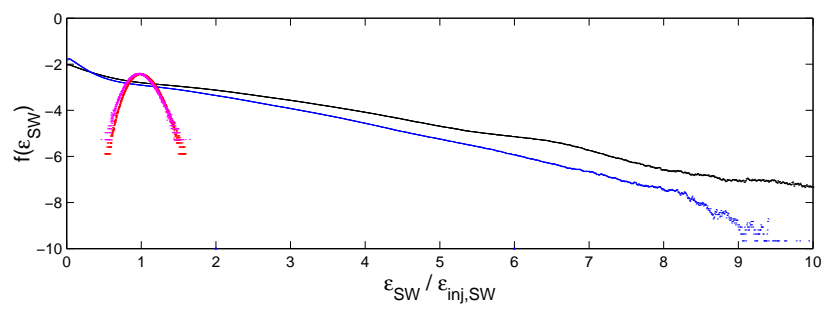

Fig. 9. Solar wind proton energy distribution functions, kinetic energy normalised to $E_{\text {inj, SW }}$ vs. normalised frequency on a $\log _{10}$ scale. Results are shown both in the absence of pickup protons (black) and with $10 \%$ pickup protons (blue). For comparison, the upstream distribution functions are also shown, in the absence of pickup protons in red and with $10 \%$ in magenta.

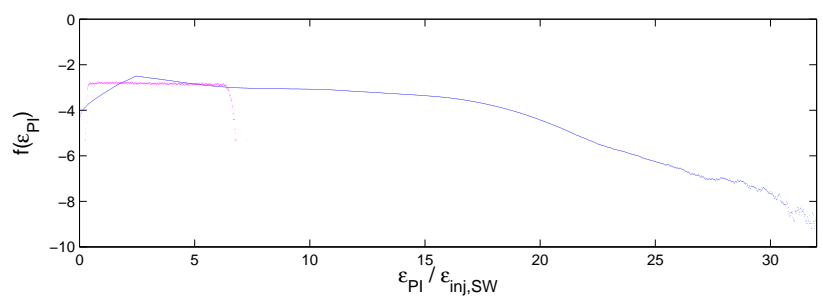

Fig. 10. Pickup proton energy distribution functions, kinetic energy

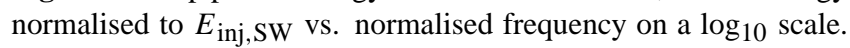
Colours are as in Fig. 9.

equal magnitude $\left(\sim \mathcal{E}_{\text {inj,Sw }}\right)$. After reflection this is followed by an $x$-energy gain, also of roughly equal magnitude, as the protons move out into the foot region. The magnitudes are similar because the protons experience similar potential structures. However, there is a slight disparity because the pickup proton interacts with the shock front and transfers some energy from its $x$-component to its $y$-component (at $t \sim 30.3 \times 2 \pi \omega_{c i}^{-1}$ ), whereas this process is absent from the solar wind proton trajectory which remains within the foot region ( $t=30.3$ to $32.8 \times 2 \pi \omega_{c i}^{-1}$ in panel 5).

Examining the $y$-components of energy (blue, panels 1 and 2 of Fig. 8), the origin of the disparity in final energies becomes clear. The pickup proton moves into the shock at a higher velocity, and is therefore reflected at that higher velocity back into the upstream region (compare panels 3 and 5 of Fig. 3). Consequently, the distance travelled by pickup protons in the foot region, upstream of the shock, is greater than that for the solar wind protons. It follows that the drift in the $y$-position is greater (panel 4), so that the gain in the $y$-component of energy, through $E_{y}$ motional drift, is greater. Examining $\int E_{x} d x$ at the particle positions (panel 3), we see that the overall potential structures around the times of $y$ energy gain are similar for both particles. Energisation occurs during a phase when the local potential is approximately constant: again, as the pickup proton travels further in this state, and as the $y$-energy gain is roughly linear, it gains more energy. As the particle passes through the foot region for a second time, the energisation finishes and the particle crosses the shock front. Consequently, solar wind protons are seen at energies up to and beyond $\sim 6 \mathcal{E}_{\text {inj. SW }}$ and pickup protons at up to $\sim 20 \mathcal{E}_{\text {inj,Sw. The self-consistent inclusion }}$ of pickup ions leads to additional downstream magnetic field structures, that can lead to further acceleration of the pickup protons, as indicated by the scattering around $x \simeq 83 \lambda_{c i}$ in the pickup proton energies in Fig. 3.

To illustrate changes in energisation mechanisms that occur due to the presence of pickup ions, the normalized distribution functions of the proton energies, $f(\mathcal{E})$, for each species are plotted on semi-log axes verses energy, normalized to the solar wind proton injection energy, in Figs. 9 and 10. The distribution functions shown here were collected over ten upstream proton gyroperiods from a region starting at $x \simeq 12 \lambda_{c i}$ downstream of the shock front, and extending for $x \simeq 5 \lambda_{c i}$. These distances are sufficiently far downstream to be in a region of strongly fluctuating fields and over spatio-temporal scales sufficiently large as to average over the small-scale variations present within the downstream populations. For comparison the distribution functions in the upstream region are also shown. These space and time averaged distributions capture the extended tail of $f(\mathcal{E})$ which cannot be seen in the snapshots of phase space (Figs. 3 and 4).

Figure 9 shows the solar wind proton distribution functions. Comparing the distribution functions of the simulations containing no pickup protons to one containing $10 \%$ pickup protons shows only minor differences. The peak in the downstream distribution functions is at zero, as the plots refer to the downstream rest frame. The distribution functions decay exponentially, with changes in exponent at $\sim 2 \mathcal{E}_{\text {inj, SW }}$, the energy gained by protons that specularly reflect form the stationary shock front (see, Lee et al., 2004a),

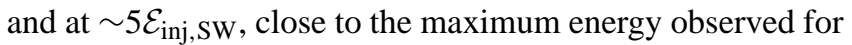
protons as they leave the shock front and enter the downstream region. Energisation can also be seen to be weaker in the presence of pickup protons.

Examining the pickup proton downstream distribution function (Fig. 10, blue), we see a peak at around $2 \mathcal{E}_{\text {inj, SW, }}$ the maximum energy of the inflowing pickup protons. The distribution function varies slowly on this $\log$ axis up to

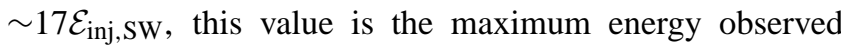
for pickup protons leaving the shock front and entering the downstream (Fig. 3, panel 3). At higher energies the distribution decays exponentially, which is suggestive of acceleration in the downstream region.

\section{Conclusions}

We have performed PIC simulations for a quasiperpendicular shock in a parameter range where the shock solutions are reforming and which is appropriate for the heliospheric termination shock. We include a shell distribution of $10 \%$ pickup protons, centred in velocity space on $v_{\text {inj }}$, with a radius $v_{1} \simeq 25 \times v_{\text {thm,SW. }}$. 
1. The addition of $10 \%$ pickup protons does not modify the solar wind proton phase space and dynamics of the reforming shock found previously (Lembège and Savoini, 1992; Shimada and Hoshino, 2000; Schmitz et al., 2002a; Lee et al., 2004a).

2. The dynamics of the energised solar wind proton population proceeds as in simulations where pickup protons are absent. Typical energies reach $\sim 6 \mathcal{E}_{\text {inj, SW }}$ and beyond, however, energisation is weaker in the presence of pickup protons.

3. A subset of the pickup protons reflect off the shock to form a weak foot region which stands upstream of the shock and is quasi-stationary.

4. The reflected pickup protons gain $\mathcal{E} \sim 20 \times \mathcal{E}_{\text {inj, SW }}$ due to this dynamics. This acts as a suprathermal population which could then act as a seed population for subsequent acceleration at the shock, which can in turn generate ACRs.

5. There is some evidence of further acceleration of pickup protons downstream of the shock ramp.

The energisation seen in our simulations takes place in a broad volume spanning the shock, its extended upstream foot, and downstream. Both the degree of energisation, and the extent of the region in which it occurs, are substantial. This implies that some of these effects may be observable, depending upon instrumental availability and resolution, and may assist the interpretation of heliopause data.

Finally, our simulations, whilst generating a suprathermal population of protons, do not directly generate ACRs. The reforming shock solutions, under the restrictions of these simulations, provide an "injection" or seed population for other mechanisms, such as diffusive Fermi acceleration (Bell, 1978; Malkov and Drury, 2001). Importantly, the reforming shock solutions have an effective reflection probability for the protons which differs from that of a static solution (Lee et al., 2004b). Whether or not a solar wind proton is reflected depends critically upon when it encounters the shock ramp, rather than where it is located in the phase space (Burgess et al., 1989, compare). This may have implications for processes such as shock surfing (as conjectured by Scholer et al., 2003) and multiple reflection (as found for static shock solutions by Zank et al., 1996; Lipatov and Zank, 1999). In this context Zank et al. (1996) have noted that the shock ramp thickness is a critical parameter. In common with previous simulations (see, for example, Shimada and Hoshino, 2000; Schmitz et al., 2002a) and with the assumption of (Zank et al., 1996), the shock ramp width in the simulation presented here is $\sim 2 c / \omega_{p e}$. However, the extension of these PIC simulations to higher dimensions, whilst retaining reforming shock solutions (Lembège and Savoini, 1992), will allow wavemodes with $k$ vectors oblique to the magnetic field rather than strictly perpendicular, as in our 1.5-D simulations. The occurrence of multiple reflection thus remains an open question.
Acknowledgements. R. E. Lee acknowledges a CASE Research Studentship from the UK Particle Physics and Astronomy Research Council in association with UKAEA, and a Warwick Postgraduate Research Fellowship from the University of Warwick. This work was also supported in part by the UK Engineering and Physical Sciences Research Council. S. C. Chapman acknowledges a Radcliffe Foundation Fellowship.

Topical Editor R. Forsyth thanks two referees for their help in evaluating this paper

\section{References}

Bell, A. R.: The acceleration of cosmic rays in shock fronts - I, Mon. Not. Royal Astron. Soc., 182, 147-156, 1978.

Birdsall, C. K. and Langdon, A. B.: Plasma physics via computer simulation, Institute of Physics publishing, 1991.

Biskamp, D. and Welter, H.: Ion heating in high-Mach-number, oblique, collisionless shock waves, Phys. Rev. Lett., 28, 410 413, 1972.

Burgess, D., Wilkinson, W. P., and Schwartz, S. J.: Ion distributions and thermalization at perpendicular and quasi-perpendicular supercritical collisionless shocks, J. Geophys. Res., 94, 87838792, 1989.

Burlaga, L. F., Ness, N. F., Stone, E. C., McDonald, F. B., Acuña, M. H., Lepping, R. P., and Connerney, J. E. P.: Search for the heliosheath with Voyager 1 magnetic field measurements, Geophys. Res. Lett., 30, 2072-2075, 2003.

Ellison, D. C., Jones, F. C., and Baring, M. G.: Direct acceleration of pickup ions at the solar wind termination shock: The production of anomalous cosmic rays, Astrophys. J., 512, 403-416, 1999.

Fisk, L. A., Kozlovsky, B., and Ramaty, R.: An Interpretation of the observed oxygen and nitrogen enhancements in low-energy cosmic rays, Astrophys. J., 190, L35-L37, 1974.

Gurnett, D. A., Kurth, W. S., and Stone, E. C.: The return of the heliospheric $2-3 \mathrm{kHz}$ radio emission during solar cycle 23 , Geophys. Res. Lett., 30, 2209-2212, 2003.

Hada, T., Onishi, B., Lembège, B., and Savoini, P.: Shock front nonstationarity of supercritical perpendicular shocks, J. Geophys. Res., 108, 1233-1244, 2003.

Hellinger, P., Trávnick, P., and Matsumoto, H.: Reformation of perpendicular shocks: Hybrid simulations, Geophys. Res. Lett., 29 , 2234-2237, 2002.

Krimigis, S. M., Decker, R. B., Hill, M. E., Armstrong, T. P. Gloeckler, G., Hamilton, D. C., Lanzerotti, L. J., and Roelof, E. C.: Voyager 1 exited the solar wind at a distance of $\sim 85 \mathrm{AU}$ from the Sun, Nature, 426, 45-48, 2003.

Kucharek, H. and Scholer, M.: Injection and acceleration of interstellar pickup ions at the heliospheric termination shock, J. Geophys. Res., 100, 1745-1754, 1995.

Lee, R. E., Chapman, S. C., and Dendy, R. O.: Numerical simulations of local shock reformation and ion acceleration in supernova remnants, Astrophys. J., 604, 187-195, 2004.

Lee, R. E., Chapman, S. C., and Dendy, R. O.: Ion acceleration processes at reforming collisionless shocks, Phys. Plasmas, 12, 102 901-102 909, 2005

Lembège, B. and Dawson, J. M.: Self-consistent study of a perpendicular collisionless and non-resistive shock, Phys. Plasmas, 30, 1767-1788, 1987.

Lembège, B. and Savoini, P.: Nonstationarity of a two-dimensional quasi-perpendicular supercritical collisionless shock by self- 
reformation, Phys. Fluids B, 4, 3533-3548, 1992.

Lembège, B. and Savoini, P.: Formation of reflected electron bursts by the nonstationarity and nonuniformity of a collisionless shock front, J. Geophys. Res., 107, 1037-1055, 2002.

Lever, E. L., Quest, K. B., and Shapiro, V. D.: Shock Surfing vs. Shock Drift Acceleration, Geophys. Res. Lett., 28, 1367-1370, 2001.

Lipatov, A. S. and Zank, G. P.: Pickup Ion Acceleration at Low- $\beta_{p}$ Perpendicular Shocks, Phys. Rev. Lett., 82, 3609-3612, 1999.

Malkov, M. A. and Drury, L. O'C.: Nonlinear theory of diffusive acceleration of particles by shock waves, Rep. Prog. Phys., 64, 429-481, 2001.

McDonald, F. B., Stone, A. C., Cummings, E. C., Heikkila, B., Lal, N., and Webber, W. R.: Enhancements of energetic particles near the heliospheric termination shock, Nature, 426, 48-51, 2003.

Pesses, M. E., Jokipii, J. R., and Eichler, D.: Cosmic ray drift, Shock wave acceleration, and the anomalous component of Cosmic Rays, Astrophys. J., 246, L85-L88, 1981.

Quest, K. B.: Simulations of High-Mach-Number Collisionless Perpendicular Shocks in Astrophysical Plasmas, Phys. Rev. Lett., 54, 1872-1874, 1985.

Rice, W. K. M., Zank, G. P., Richardson, J. D., and Decker, R. B.: Ion injection and shock acceleration in the outer heliosphere, Geophys. Res. Lett., 27, 509-512, 2000.
Schmitz, H., Chapman, S. C., and Dendy, R. O.: The influence of electron temperature and magnetic field strength on cosmic ray injection in high Mach number shocks, Astrophys. J., 570, 637646, 2002a.

Schmitz, H., Chapman, S. C., and Dendy, R. O.: Electron Preacceleration Mechanisms in the Foot Region of High Alfvénic Mach Number Shocks, Astrophys. J., 579, 327-336, 2002 b.

Scholer, M., Shinohara, I., and Matsukiyo, S.: Quasi-perpendicular shocks: Length scale of the cross-shock potential, shock reformation, and implication for shock surfing, J. Geophys. Res., 108, 1014-1024, 2003.

Shimada, N. and Hoshino, M.: Strong electron acceleration at high Mach number shock waves: Simulation study of electron dynamics, Astrophys. J., 543, L67-L71, 2000.

Smith, E. J.: Magnetic fields throughout the heliosphere, Adv. Space Res., 13, (6)5-(6)14, 1993.

Zank, G. P., Pauls, H. L., Cairns, I. H., and Webb, G. M.: Interstellar pickup ions and quasi-perpendicular shocks: Implications for the termination shock and interplanetary shocks, J. Geophys. Res., 101, 457-477, 1996.

Zank, G. P., Rice, W. K. M., Le Roux, J. A., and Matthaeus, W. H.: The injection problem for Anomalous Cosmic Rays, Astrophys. J., 556, 494-500, 2001. 